\title{
An equilibrium approach to international merger policy
}

\author{
Luís M.B. Cabral* \\ New York University, United States \\ CEPR, United Kingdom
}

Available online 26 September 2005

\begin{abstract}
I treat international merger policy as a repeated veto game. I show that there exists a unique efficient equilibrium within a particular class of trigger strategy equilibria. I then consider a series of comparative statics and extensions: (a) if for some exogenous reason one of the countries becomes more lenient towards mergers, than the other country becomes more lenient as well; (b) merger remedies increase the probability that a merger is approved and increase total welfare; (c) the effects of a merger wave are magnified by the equilibrium approval policy.

(C) 2005 Elsevier B.V. All rights reserved.
\end{abstract}

JEL classification: F0; L4

Keywords: Merger policy; Repeated games

\section{Introduction}

In a world that is increasingly global, merger policy seems to follow the trend. The GE/ Honeywell merger and other related cases show that large mergers must be approached from an international perspective. They also show that different merger authorities may have different evaluations of the welfare impact of a merger, different utility functions, or both. Whichever is the case, the fact is that any proposed merger of significant size must pass at least the U.S. and the EU tests.

As a solution to the problem of merger policy in a global world, former U.S. Assistant Attorney General Joel Klein proposed the creation of a world wide merger

* Stern School of Business, 44 West 4th Street, New York, NY 10012, United States.

E-mail address: lcabral@stern.nyu.edu. 
authority. ${ }^{1}$ However, this solution is unlikely to be feasible, both politically and informationally. Politically, the problems stem from the near impossibility of enforcement at the international level. Informationally, there is the problem inherent in centralized decision making. ${ }^{2}$

In this paper, I propose a solution to the enforcement problem. Specifically, I propose the equilibrium self-enforcement of merger policy in a repeated interaction context. The idea of using repeated interaction to enforce cooperative agreements is obviously not novel. However, the specific nature of the game played between antitrust authorities warrants a specific analysis. I characterize an efficient trigger strategy Nash equilibrium and show that it is unique (within its class). In this equilibrium, each country is willing to accept a merger proposal that is welfare reducing from that country's perspective but welfare increasing from a worldwide perspective.

I consider a number of extensions of the basic framework. In Section 5, I study the case when one of the countries becomes more lenient towards mergers and show that, under some conditions, this leads the other country to be more lenient as well ("contagious leniency"). In Section 6, I consider the possibility of merger remedies, which I model as welfare reducing utility transfers. Finally, in Section 7 I study the impact of a merger wave. I show that the frequency of approved mergers increases during the wave, so that the equilibrium policy magnifies the effects of the merger wave.

\section{Repeated merger policy games}

Over time, a number of merger proposals take place, some among firms in a given country, some among firms from different countries. Whichever is the case, the welfare impact of a specific merger is likely to be different in different countries. In addition to differences in location, the impact of the merger may differ because different countries place different weights on profits and consumer surplus. ${ }^{3}$ Given a merger proposal, antitrust authorities in each country must decide whether or not to approve the merger.

I model this situation as a repeated game between two countries. In each period of the repeated game, Nature determines whether a merger is proposed (probability $\rho$ ). Nature also determines the welfare impact of the merger in country $i, w_{i}(i=1,2)$, according to the c.d.f. $F\left(w_{1} ; w_{2}\right)$, which I assume is smooth and has full support. If a merger is proposed, then each country's merger authority decides whether to approve the merger. Finally, I make the important assumption that a merger only takes place if approved by both merger authorities.

Consider the stage game where a merger is proposed and the values of $w_{i}$ are observed. A natural equilibrium of this game is for the merger to go through if and only if $w_{i} \geq 0$ for both $i$. The problem with this equilibrium is that many efficient mergers are vetoed. Fig. 1 depicts this problem. Let $S$ be the set of possible values of $\left(w_{1}, w_{j}\right)$. Efficient mergers correspond to points to the NE of the second diagonal, $w_{1}+w_{2} \geq 0$. This area can be subdivided into three subregions. In region $\mathrm{A}$, the merger is welfare improving for both countries. In region D1, the merger increases Country 2's welfare but decreases Country 1's welfare (by a lower amount). Finally, in region D2 the opposite is true: Country 1's welfare increases but Country 2's decreases (by a lower

\footnotetext{
1 "I...believe that, whatever happens on antitrust at the WTO..., we should move in the direction of a Global Competition Initiative, cautiously and on an exploratory basis, but in the end I think such a development is almost inevitable" (Klein, 2000). For a different perspective, see Fox (1998).

${ }^{2}$ In this regard, see Barros and Cabral (1994), Neven et al. (1994), Bacchetta et al. (1997), Head and Ries (1997), Neven and Röller (2000).

${ }^{3}$ Barros and Cabral (1994), Neven and Röller (2001) and others develop models that suggest possible sources of divergence across antitrust authorities. I take a reduced-form approach that is consistent with all of these models.
} 


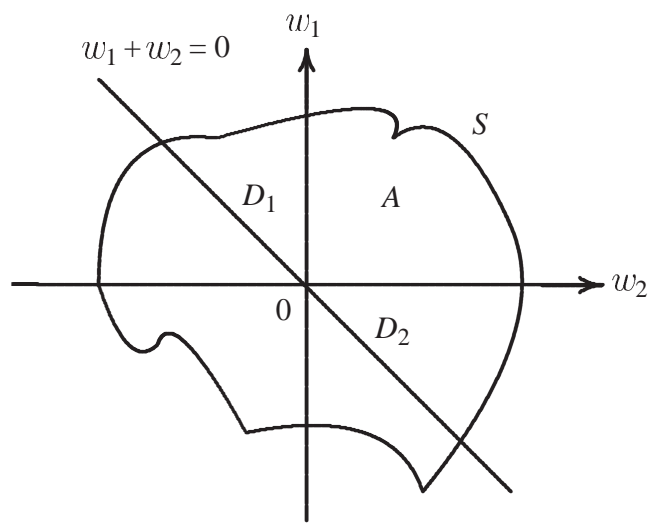

Fig. 1. Agreement and disagreement over merger decisions.

amount). In the static equilibrium considered above, only mergers in Region A go through. Mergers lying in Regions $D_{i}$, though efficient, are not approved.

Consider now the infinite repetition of the above stage game, making the additional assumptions that the values of $w_{i}$ are independently distributed across periods and that both countries have a common interest rate $r$. Define $\delta \equiv \rho / 1+r$ the effective discount factor. ${ }^{4}$ The basic intuition from repeated game theory suggests that the set of attainable payoffs in the repeated game is larger than the set of stage equilibria. In other words, there are mergers that would not be approved in a static equilibrium but might be approved in a dynamic equilibrium. The intuition is that each country will refrain from pursuing its short-run interest as this might reduce future payoff. I make this point more precise in the next section.

\section{Maximal concession equilibria}

Consider a set of equilibrium strategies $x_{i}^{t}\left(w^{t}, h^{t}\right), i=1,2$ indicating the probability that country $i$ approves the proposal submitted at time $t, w^{t}=\left(w_{1}^{t}, x_{2}^{t}\right)$, given a history $h^{t}$ of past decisions by both countries. I focus on the set of optimal trigger-strategy equilibria that maximize joint discounted payoff, $\sum_{t=0}^{\infty} \int_{S} \delta^{t} x_{1}^{t}\left(w^{t}, h^{t}\right) x_{2}^{t}\left(w^{t}, h^{t}\right)\left(w_{1}^{t}+w_{2}^{t}\right) d F(w)$, subject to the constraint that the strategies $x_{1}^{t}\left(w^{t}, h^{t}\right)$ form a subgame perfect Nash equilibrium in trigger strategies with reversion to the static Nash equilibrium. A trigger strategy equilibrium is defined by a cooperative phase, a punishment phase, and the rules that: (a) play starts in the cooperative phase; (b) play continues in the cooperative phase as long as players choose the designated cooperative action; (c) play switches to the start of the punishment phase whenever a player chooses an action different from the designated action for whatever phase play is on; (d) starting from the punishment phase, play reverts to the cooperative phase after $T$ periods. A triggerstrategy equilibrium with reversion to Nash corresponds to the case when the punishment phase amounts to the play of the static Nash equilibrium. For simplicity, I will refer to the above optimal equilibria as simply "optimal equilibria."

These equilibria correspond roughly to the equilibria developed in Green and Porter (1984) in the context of oligopoly collusion. Unlike Green and Porter (1984), I do not consider the case of incomplete information. For this reason, the optimal trigger-strategy equilibrium would

\footnotetext{
${ }^{4}$ That is, $\delta$ reflects both the length between periods and the probability that a merger proposal arises in each period.
} 


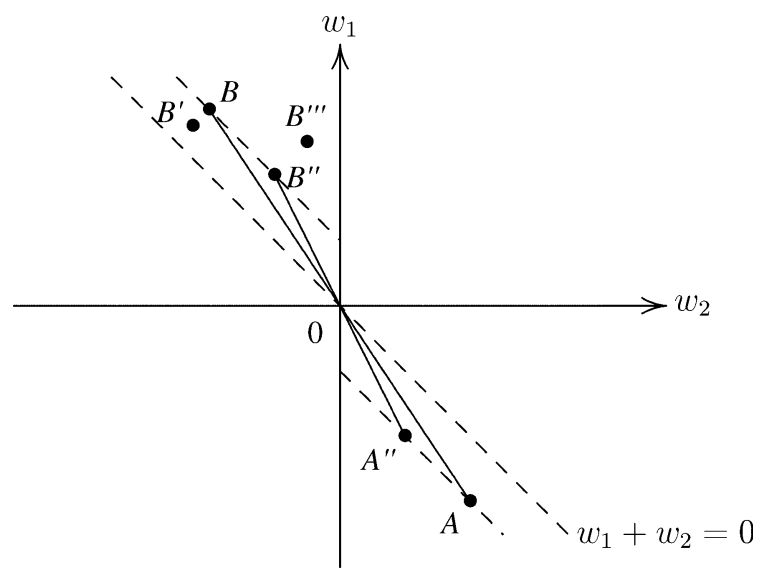

Fig. 2. Agreement and disagreement over merger decisions.

correspond to $T=\infty .^{5}$ However, a finite $T$ may be more realistic in the present context. Moreover, as will become clear, the results do not depend on the value of $T$.

My first result characterizes the structure of optimal equilibria.

Proposition 1. (maximum concession equilibria)

Along the cooperative phase of an optimal equilibrium, a merger proposal is approved by both countries if and only if (i) $w_{1}+w_{2} \geq 0$, (ii) $w_{1} \geq-l_{1}$, and (iii) $w_{2} \geq-l_{2}$.

Proof. Denote by $S$ is the set of proposals that are approved along the cooperative phase. Define $l_{i}(S)$ to be the highest loss that country $i$ can sustain such that the no-deviation constraint in a trigger-strategy equilibrium is just satisfied. By vetoing today's merger, a country expects a discounted payoff of $0+\delta N_{i}$, where $N_{i}$ is the expected discounted payoff following deviation (history independent in the case of a trigger-strategy equilibrium). By approving the merger, a country expects a discounted payoff of $-l_{i}+\delta E_{i}$, where $E_{i}=\sum_{t=0}^{\infty} \delta^{t} \int_{S} x_{1}^{t}\left(w^{t}, h^{t}\right) x_{2}^{t}\left(w^{t}, h^{t}\right) w_{i}^{t} d$ $F(w)$ is expected payoff along the equilibrium path (assuming $x_{1}^{t}\left(w^{t}, h^{t}\right)$ is the equilibrium strategy described in the proposition). We thus have $l_{i}(S)=\delta\left(E_{i}-N_{i}\right)$.

The proof has two parts. First I show necessity, viz. that if $w \in S$ then (i) $w_{1}+w_{2} \geq 0$, (ii) $w_{1} \geq-l_{i}$ and (iii) $w_{2} \geq-l_{2}$. Second I show sufficiency, viz. that if (i) $w_{1}+w_{2} \geq 0$, (ii) $w_{1} \geq-l_{i}$ and (iii) $w_{2} \geq l_{2}$.then $w \in S$.

Part A: necessity. I first prove that $w_{1}+w_{2} \geq 0$ must hold for all points in $S$. Refer to Fig. 2 . Suppose that $w_{i}^{A} \geq l_{i}$, but $w_{1}^{A}+w_{2}^{A}<0$. Without loss of generality, suppose also that $w_{1}^{A}<0$. (If there is more than one such point satisfying the above conditions, select the one with the lowest $w_{2}$ ). Consider the points along the line containing the origin and point $A$. Suppose there exists a point $B$ along the same diagonal, in the opposite quadrant, that also belongs to $S$. Consider a ball around $A, \mathcal{B}(A)$; and one around $B ; \mathcal{B}(B)$; with densities such that

$$
-w_{1}^{A} \int_{\mathcal{B}(A)} f(w) d w=w_{1}^{B} \int_{\mathcal{B}(B)} f(w) d w .
$$

\footnotetext{
${ }^{5}$ Moreover, the optimal punishment would be the extremal punishment. If such punishment were played, then the optimal trigger-strategy equilibrium would be globally optimal among the set of subgame perfect Nash equilibria.
} 
Note that, since $A$ and $B$ are on the same diagonal, it must also be the case that $w_{2}^{A} \int_{\mathcal{B}(A)} f(w) d w=-w_{2}^{B} \int_{\mathcal{B}(B)} f(w) d w$.

Consider now an alternative set $S^{\prime}$ that is given by $S$ minus $\mathcal{B}(A) \cup \mathcal{B}(B)$. By construction, expected payoff in $S^{\prime}$ is the same as in $S$. Suppose then that we consider the same operation but using point $B^{\prime \prime}$ instead of point $B$, where $B^{\prime \prime}$ lies to the SW of $B$. Since $B \in S$, there must exist such a $B^{\prime \prime}$ that is also in $S$. It follows that the $S^{\prime \prime}$ set resulting from this alternative procedure yields a higher expected payoff (for both players) than $S$.

Suppose now that there exists no point $B$ along the same diagonal, in the opposite quadrant to $A$, that also belongs to $S$. Consider now a point $B$ along the same diagonal, in the opposite quadrant to $A$, such that $\mathrm{w}_{1}^{B} \geq-l_{1}$.

Consider also points $A^{\prime \prime}, B^{\prime \prime}$, as in the figure: $A^{\prime \prime}$ and $A$ belong to a line of slope -1 , likewise for $B$ and $B^{\prime \prime}$. Moreover, both $A^{\prime \prime}$ and $B^{\prime \prime}$ belong to the same ray that crosses the origin. If $B^{\prime \prime}$ is sufficiently close to $B$, then neither $A$ nor $B$ belong to $S$. Consider an alternative set $S^{\prime}$ obtained from $S$ by adding balls around $A^{\prime \prime}$ and $B^{\prime \prime}$ with mass such that Eq. (1) holds (for points $A^{\prime \prime}$ and $B^{\prime \prime}$ instead of $A, B$ ). Expected payoff is the same in $S^{\prime}$ as in $S$. But then we can construct a new set $S^{\prime \prime}$ with a point $B^{\prime \prime \prime}$ slightly to the NE of $B^{\prime \prime}$, yielding a higher expected payoff for both players.

To conclude part A of the proof, note that $w_{i} \geq l_{i}(S)$ is also necessary. In fact, I defined $l_{i}(S)$ as the value such that the no-deviation constraint is exactly satisfied.

Part B: sufficiency. I now show that, (i) if $w_{1}+w_{2} \geq 0$, (ii) $w_{1} \geq-l_{1}$, and (iii) $w_{2} \geq-l_{2}$, then in an optimal trigger-strategy equilibrium it must be that $w \in S$. Consider a point $A$ not in, but close to, $S$. Suppose that $w_{1}^{A}>-l_{1}, w_{1}^{B}>-l_{1}, w_{1}^{A}+w_{1}^{B}>0$. Suppose there is a point $B \in S$ in the same quadrant as $A$ and such that $w_{1}^{B}+w_{2}^{B}=w_{1}^{A}+w_{2}^{A}, w_{1}^{B}>-l_{1}, w_{2}^{B}>-l_{2}$. We can then find balls $\mathcal{B}(A)$ and $\mathcal{B}(B)$ such that adding $\mathcal{B}(A)$ to and subtracting $\mathcal{B}(B)$ from $S$ keeps both players' expected payoff constant. But then there must exist a point $B^{\prime \prime}$ to the $\mathrm{SW}$ of $B^{\prime}$ such that adding $\mathcal{B}(A)$ to and subtracting $\mathcal{B}\left(B^{\prime}\right)$ from $S$ increases both players' payoff, which contradicts optimality.

Suppose now that no point $B$ exists satisfying the above conditions. Then there exists an $A^{\prime} \in S$ (close to $A$ ) and a $B^{\prime \prime} \notin S$ in the same quadrant and such that $w_{1}^{B^{\prime \prime}}+w_{2}^{B^{\prime \prime}}=w_{1}^{A^{\prime}}+w_{2}^{A^{\prime}}$, $w_{1}^{B^{\prime \prime}}>-l_{1}, w_{2}^{B^{\prime \prime}}>-l_{2}$. We can then find balls $\mathcal{B}\left(A^{\prime}\right)$ and $\mathcal{B}\left(B^{\prime \prime}\right)$ such that adding $\mathcal{B}\left(B^{\prime \prime}\right)$ to and subtracting $\mathcal{B}\left(A^{\prime}\right)$ from $S$ keeps both players' expected payoff constant. But then there must exist a point $A^{\prime \prime}$ to the $\mathrm{SW}$ of $A^{\prime}$ such that adding $\mathcal{B}\left(B^{\prime \prime}\right)$ to and subtracting $\mathcal{B}\left(A^{\prime}\right)$ from $S$ increases both players' payoff, which contradicts optimality.

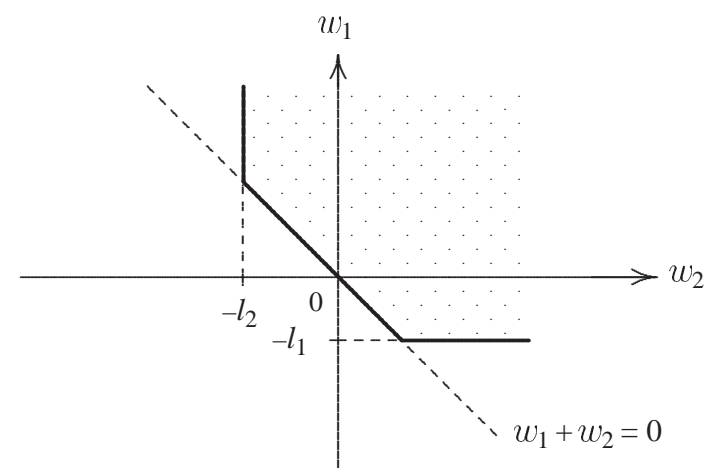

Fig. 3. Optimal equilibrium: proposed mergers in the shaded region are approved (along the equilibrium path). 
Proposition 1 is in line with well-known results in the repeated-game literature. The novel aspect here is the precise nature of the cooperative phase, namely the fact that each country approves efficient mergers up to a maximum "concession" level $-l_{i}$. In words, each country is willing to accept a negative welfare impact $-l_{\mathrm{i}}$ in the short run in return for the other country not vetoeing future efficient mergers where country $i$ stands to gain but country $j$ loses (by a smaller amount). Fig. 3 illustrates the result. In this figure, the mergers belonging to the shaded region are approved along the equilibrium path. Notice that, if $\delta$ is less than one and $S$ is sufficiently large, then there will still be efficient mergers that are not approved in equilibrium. For a finite $S$, it can be shown that there exists a $\bar{\delta}$ such that, if $\delta>\bar{\delta}$, then all efficient mergers are approved in equilibrium (folk theorem).

In the next three sections, I consider some extensions of the optimal equilibrium when $\delta<\bar{\delta}$.

\section{Uniqueness}

I now show that there exists a unique optimal equilibrium, that is, a unique equilibrium in trigger strategies with reversion to Nash that maximizes joint payoffs. ${ }^{6}$ This result is interesting from a game-theoretic point of view. More importantly, its proof suggests an important property: the "complementarity" between each country's value of $l_{i}$.

Proposition 2. (uniqueness)

There exists a unique optimal equilibrium.

Proof. Consider a trigger-strategy equilibrium with reversion to the static Nash equilibrium. The no-deviation constraint is given by

$$
-l_{i}+\delta E_{i} \geq \delta N_{i}
$$

or simply

$$
l_{i} \leq \Phi_{i}\left(l_{i}, l_{j}\right),
$$

where

$$
\Phi_{i}\left(l_{i}, l_{i}\right) \equiv \frac{\delta}{1-\delta}\left(\int_{w_{i}>-l_{i}} w_{i} f(w) d w-\int_{w_{i}>0} w_{i} f(w) d w\right) .
$$

Notice that (a) $\Phi_{i}(0,0)=0$, (b) $\Phi_{i}\left(0, l_{j}\right) \geq 0$ (with strict inequality if $l_{j}>0$ ), (c) $\Phi_{i}\left(l_{i}, l_{j}\right)$ is bounded, (d) $\Phi_{i}\left(l_{i}, l_{j}\right)$ is strictly decreasing in $l_{i}$, (e) $\Phi_{i}\left(l_{i}, l_{j}\right)$ is strictly increasing in $l_{j}$. This implies that (a) there exists a unique $l_{i}^{*}\left(l_{j}\right)$ such that $l_{i}=\Phi_{i}\left(l_{i}, l_{j}\right),(\mathrm{b}) l^{*}(0)=0$, (c) $l_{i}^{*}\left(l_{j}\right)$ is strictly increasing in $l_{j}$, (d) $l_{i}^{*}\left(l_{j}\right)$ is bounded.

The above imply that the set of feasible equilibrium values $l_{i}$, defined by $l_{i} \leq l_{i}^{*}\left(l_{j}\right)$, must have a shape like that in Fig. 4. It follows that for any utility function increasing in $l_{i}$ there exists a unique optimal value. The extension to the case of $T$ period punishments is straightforward.

In words, the essence of the proof of Proposition 2 is that there is a "complementarity" between the values of $l_{i}$. That is, the maximum concession mappings $l_{i}^{*}\left(l_{j}\right)$ are increasing. In the

\footnotetext{
${ }^{6}$ In fact, as will become clear, the equilibrium is unique for any joint welfare function $W$ such that $\left(\partial W / \partial w_{i}\right)>0$.
} 


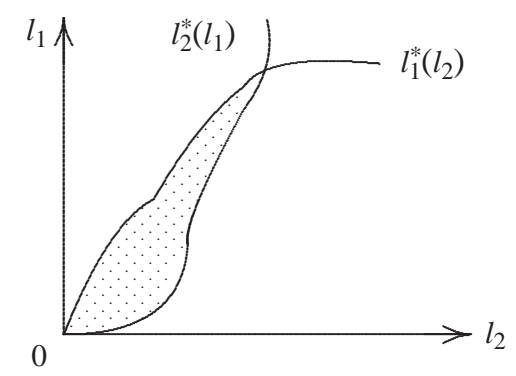

Fig. 4. Set of feasible equilibrium values $\left(l_{1}, l_{2}\right)$.

next section, I elaborate on this interesting fact and show that, under some conditions, an increase in leniency by one country leads to an increase in leniency by the other country as well.

\section{Contagious leniency}

Suppose that one of the countries changes its utility function. Specifically, I consider the case when one of the countries becomes more lenient towards mergers. For example, if a country places an increasing relative weight on firm profits, then the welfare impact of the merger is likely to be higher (in other words, for a given merger the value of $w_{i}$ is higher). I model "increased leniency" by assuming that the distribution of $w$ is given by $F\left(w_{1}-\alpha, w_{2}\right)$ and considering an increase in $\alpha$ (that is, Country A becoming more lenient). The main result is that, under some conditions, an increase in leniency by one of the countries leads both countries to increase their maximum concession levels $l_{i}$. That is, in terms of optimal equilibrium strategies, leniency is "contagious." For the purpose of the next result, it is useful to define $\underline{\delta}$ to be the infimum of all values of $\delta$ such that a non-trivial optimal equilibrium $\left(l_{i}>0\right)$ is viable.

Proposition 3. (contagious leniency)

Suppose that $\delta$ is greater than, but close to, $\underline{\delta}$. Then $\partial l_{i} / \partial \alpha>0, i=1,2$. In words, as a country becomes more lenient towards mergers (increase in $\alpha$ ) both countries increase their levels of mutual concession $\left(-l_{i}\right)$.

Proof. Let

$$
\Phi_{i}\left(l_{i}, l_{j} ; \alpha, \delta\right) \equiv \frac{\delta}{1-\delta}\left(\int_{w_{i}>-l_{i}} w_{i} f(w ; \alpha) d w-\int_{w_{i}>0} w_{i} f(w ; \alpha) d w\right) .
$$

In a optimal equilibrium, $l_{i}=\Phi_{i}\left(l_{i}, l_{j} ; \alpha, \delta\right)$. Differentiating with respect to $\alpha$, we get

$$
\left[\begin{array}{cc}
1-\frac{\partial \Phi_{1}}{\partial l_{1}} & -\frac{\partial \Phi_{1}}{\partial l_{2}} \\
-\frac{\partial \Phi_{2}}{\partial l_{1}} & 1-\frac{\partial \Phi_{2}}{\partial l_{2}}
\end{array}\right]\left[\begin{array}{l}
d l_{1} \\
d l_{2}
\end{array}\right]=\left[\begin{array}{c}
\frac{\partial \Phi_{1}}{\partial \alpha} \\
\frac{\partial \Phi_{2}}{\partial \alpha}
\end{array}\right] d \alpha
$$

Solving with respect to $l_{i}$, we get

$$
\frac{d l_{i}}{d \alpha}=\Delta^{-1}\left(\frac{\partial \Phi_{i}}{\partial \alpha}\left(1-\frac{\partial \Phi_{j}}{\partial l_{j}}\right)+\frac{\partial \Phi_{j}}{\partial \alpha} \frac{\partial \Phi_{i}}{\partial l_{j}}\right)
$$


where

$$
\Delta \equiv\left(1-\frac{\partial \Phi_{1}}{\partial l_{1}}\right)\left(1-\frac{\partial \Phi_{2}}{\partial l_{2}}\right)-\frac{\partial \Phi_{1}}{\partial l_{2}} \frac{\partial \Phi_{2}}{\partial l_{1}}>0 .
$$

Let $\delta$ be the infimum of all values of $\delta$ such that a non-trivial optimal equilibrium $\left(l_{i}>0\right)$ is viable. Suppose that $\delta=\underline{\delta}+\epsilon$, where $\epsilon$ is arbitrarily small. It follows that the values $l_{i}$ in the optimal equilibrium are of order $\epsilon$ too.

I will now consider a shift in $F$ along Player 1's axis and argue that $\partial \Phi_{1} / \partial \alpha$ is of greater order of magnitude than $\partial \Phi_{2} / \partial \alpha$. Recall that $\Phi_{i}$ is proportional to the difference between expected payoff along the equilibrium path and expected payoff along the one-shot Nash equilibrium. This difference corresponds to areas $A_{1}$ and $A_{2}$ in Fig. 5.

For Player 1, the change in $F_{1}$ originating in $A_{1}$ is of order $\epsilon d \propto$, where $\epsilon$ comes from the fact that the probability of being in area $A_{1}$ is of order $\epsilon$. The change in $F_{1}$ originating in $A_{2}$ is of order $\epsilon^{3} d \propto$, where one $\epsilon$ comes from the probability of being in $A_{2}$, one from the value of $w_{1}$ (being of order $\epsilon$ ), and one from the change in $f(w)$ (also of order $\epsilon$ ).

For Player 2, the change in $F_{2}$ originating in $A_{2}$ is of order $\epsilon^{2} d \alpha$, where one $\epsilon$ comes from the probability of being in area $A_{1}$ and the other from the value of $w_{2}$. The change in $\Phi_{2}$ originating in $A_{2}$ is of order $\epsilon^{2} d \propto$, where one $\epsilon$ comes from the probability of being in $A_{2}$ and the other from the change in $f(w)$.

From Eq. (2), we then get

$$
\operatorname{sign}\left(\frac{d l_{i}}{d \alpha}\right)=\operatorname{sign}\left(\frac{\partial \Phi_{j}}{\partial \alpha} \frac{\partial \Phi_{i}}{\partial l_{j}}\right)>0,
$$

since both $\partial \Phi_{j} / \partial \alpha$ and $\partial \Phi_{j} / \partial l_{j}$ are positive.

The intuition for Proposition 3 can be seen with reference to Fig. 4. An increase in $\alpha$ implies an outward shift in $l_{1}^{*}\left(l_{2}\right)$. If $l_{2}^{*}\left(l_{1}\right)$ does not change that much, then the shift in $l_{1}^{*}\left(l_{2}\right)$ will result in an increase both in $l_{1}$ and in $l_{2}$. In other words, Proposition 3 results from the fact that $l_{2}^{*}\left(l_{1}\right)$ is increasing. The assumption that $\delta$ is greater than, but close to, $\delta$ is quite important. For higher values of $\delta$, both $l_{1}^{*}\left(l_{2}\right)$ and $l_{2}^{*}\left(l_{1}\right)$ will shift with a change in $\alpha$. Intuitively, the fact that $E_{2}$ increases makes Country 2 more willing to be lenient (increase in $l_{2}$ ). However, an increase in $\alpha$ also increases $N_{2}$. It is not generally clear which effect will dominate. In a separate paper, Cabral (2003), I show by numerical example that, if $F$ is uniform and for particular parameter values,

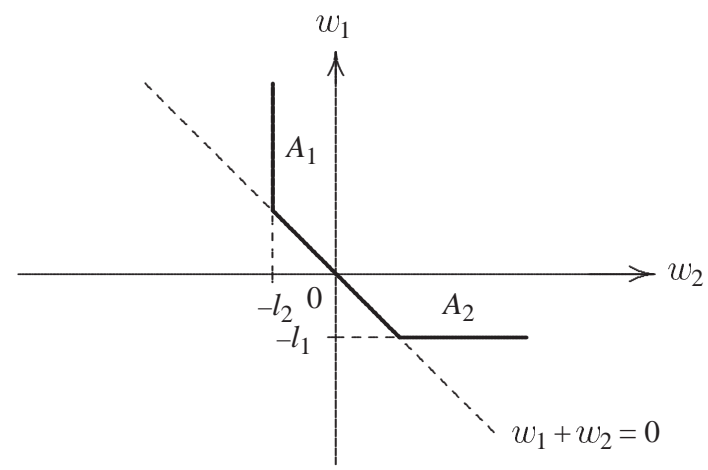

Fig. 5. Relevant areas for variation in $\Phi_{i}$ caused by changes in $\alpha$. 
$\partial l_{2} / \partial \alpha$ is positive for low values of $\alpha$ but negative for high values of $\alpha$. When $\partial l_{2} / \partial \alpha$ is negative, the effect on $N_{2}$ dominates the effect on $E_{2}$.

\section{Remedies}

In the previous sections, I have made the assumption that merger proposals are exogenously given and that there is nothing merger authorities can do about it other than to decide whether to approve it or not. Clearly, things are more complex in practice. Specifically, it is frequently the case that mergers are conditionally approved. For example, in the recent GE/Honeywell merger proposal, the EU requested a series of asset sales as a condition for giving its approval (eventually, GE refused the offer and the EU blocked the merger).

I model the possibility of asset sales or other remedies by changing the structure of the stage game. In the previous sections, I have assumed that once a merger proposal $w$ is exogenously given, each country simultaneously approves or vetoes the merger. Now I suppose that, if $w_{i}<0$, then country $i$ has the option of requesting a change in the terms of the merger as a condition for its approval.

The typical request by country $i$ would be some form of asset transfer. Naturally, such operation would imply a change in welfare impact both for country $i$ and for country $j$. Accordingly, I model the possibility of merger remedies by assuming that utility can be transferred across countries according to $\Psi\left(w^{\prime} ; w\right)=0$. In words, starting from merger proposal $w$, all points $w^{\prime}$ such that $\Psi\left(w^{\prime} ; w\right)=0$ are attainable by means of merger remedies.

Typically, the implicit plot of $\Psi\left(w ; w^{\prime}\right)=0$ will have a slope lower than one: in order to increase country $i$ 's welfare by one dollar, country $j$ 's welfare is decreased by more than a dollar. For example, GE's asset divesture as part of the Honeywell deal would increase EU's welfare (given its impact on GE's European competitors and, possibly, European consumers). However, its impact on GE's profit is likely to have a greater negative impact on US welfare than the positive impact in the EU.

My main result in this section is that the possibility of asset sales increases the set of approved mergers.

\section{Proposition 4. (remedies)}

Suppose that $F$ is symmetric about the main diagonal. Along the cooperative phase of an optimal equilibrium, both countries approve a merger (with no remedies) if $w_{1}+w_{2} \geq 0$ and $w_{i} \geq-l_{i}, i=1,2$. If $w_{i}<-l_{i}$ and there exists a $w^{\prime}$ such that $\Psi\left(w^{\prime} ; w\right)=0$ and $w_{i}{ }^{\prime}=-l_{i}$, then player $j$ proposes $w^{\prime}$ and both countries approve the merger. Otherwise, the merger is vetoed by both players.

Proof. Clearly, the points in the shaded region of Fig. 3 should be in the approved-merger region with remedies. (The possibility of remedies cannot make things worse off). Consider a point $A$ that belongs to the shaded region in Fig. 6 but not to the shaded region of Fig. 3. Consider a symmetric equilibrium and suppose the merger $w_{A}$ is not approved in equilibrium. Consider an alternative equilibrium such that both $A$ and its symmetric counterpart, $A^{\prime}$ are approved. By construction, there exist points $B ; B^{\prime}$ such that (i) $\Psi(B, A)=0, \Psi\left(B^{\prime}, A^{\prime}\right)=0$; (ii) $w_{1}^{B} \geq 0, w_{1}^{B^{\prime}} \geq 0$; (iii) $w_{2}^{B} \geq 0, w_{2}^{B^{\prime}} \geq 0$; (iv) $w_{1}^{B}+w_{2}^{B} \geq 0, w_{1}^{B^{\prime}}+w_{2}^{B^{\prime}} \geq 0$. It follows that the new proposed solution (a) is a subgame perfect equilibrium, (b) yields a higher payoff.

Fig. 6 illustrates the point. In this figure, I assume, for simplicity, that the implicit plot of $\Psi\left(w^{\prime}, w\right)=0$ is linear. It should be noted that the equilibrium values of $l_{i}$ in Proposition 4 are not 


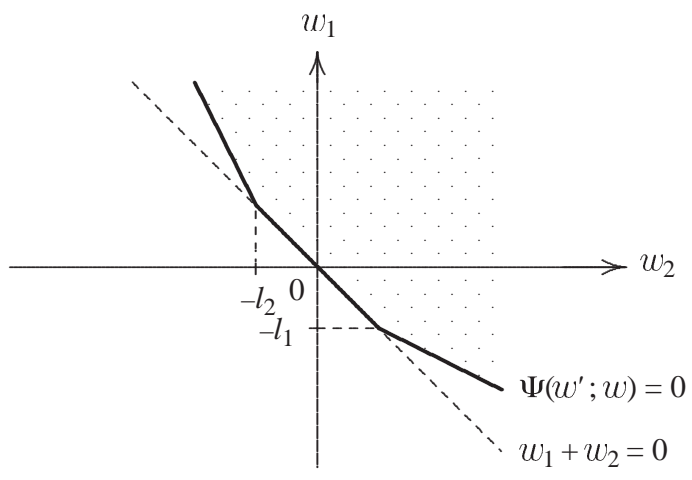

Fig. 6. Optimal equilibrium with asset sales.

identical to the ones in Proposition 1; in fact, they are greater. Finally, notice that, if the slope of $\Phi$ is greater than one, then the set of approved mergers would be even greater. In fact, there might be mergers initially classified as inefficient $\left(w_{1}+w_{2}<0\right)$ which become efficient once asset sales are taken into consideration.

\section{Merger waves}

One of the most commonly known facts about mergers is that they take place in waves. ${ }^{7}$ In terms of my model, a merger wave can be thought of as a temporary increase in $\rho$, the probability that, in each period, a merger proposal is made. My last results pertain to the dynamics of $l_{i}$ with respect to a temporary change (e.g., increase) in $\rho$. Specifically, suppose the rate of merger proposals follows a two-state Markov process (high and low). I will consider two extreme possibilities, unanticipated and anticipated merger waves, and show that both lead to the following result.

\section{Proposition 5. (merger waves)}

The fraction of approved merger proposals is higher during a merger wave than after.

Proof. Let us first consider the simplest case, unanticipated merger waves. By analogy with the previous results, let $\Phi(s)$ be the difference in discounted future profits between cooperating and deviating today, where $s$ is the current state. Notice that $\Phi(H)>\Phi(L)>0$. In fact, during the $H$ state, we could replicate the solution during the $L$ state (veto some of the mergers with some probability). Since in an optimal equilibrium $l_{i}(s)=\Phi_{i}(s)$, it follows that $l_{i}$ is higher in the high state. Finally, it follows that the probability a merger is approved is higher too.

Consider now the case of a one-time anticipated merger wave. In particular, suppose that it is known the state will switch from $L$ to $H$ from $t^{\prime}$ to $t^{\prime \prime}$ (and back to $L$ after $t^{\prime \prime}$ ). Let $\Phi(t)$ be the difference in discounted future profits between cooperating and deviating at date $t$. $\Phi(t)$ is lowest for $t>t^{\prime \prime}$, implying the fraction of proposed mergers is lowest during that period. As we move backwards from $t^{\prime \prime}$, the fraction of approved mergers increases. At $t^{\prime \prime}$, this results from a higher $\rho$. At $t^{\prime \prime}-1$, this results both from at higher $\rho$ and a higher expected future payoff at $t^{\prime \prime}$. And so

\footnotetext{
${ }^{7}$ In this paper, I take merger waves as exogenously given. For a theory of merger waves and a review of the relevant literature, see Toxvaerd (2002).
} 


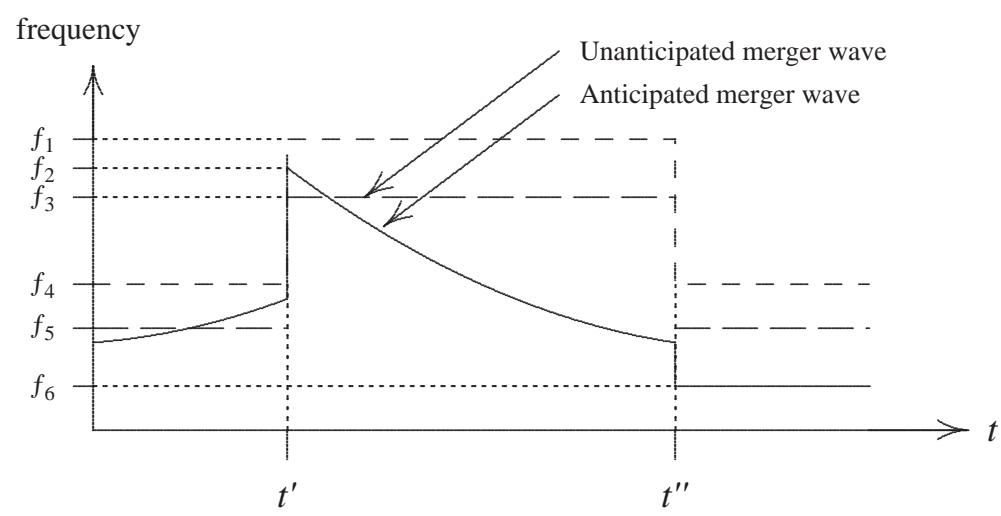

Fig. 7. Frequency of proposed mergers (short dashes) and approved mergers when (a) a merger wave in perfectly anticipated (solid line), (b) a merger wave is unanticipated (long dashes).

forth. Finally, for $t<t^{\prime}$, future expected payoff declines as we move backward in time, both because we have a lower $\rho$ and because the period of higher payoff $\left(\left[t^{\prime}, t^{\prime \prime}\right]\right)$ moves farther into the future.

Fig. 7 illustrates Proposition 5. In this figure, the frequency of proposed mergers increases during the period $\left[t^{\prime}, t^{\prime \prime}\right]$. If the merger wave in unanticipated, then the proportion of mergers that are approved increases during the period $\left[t^{\prime}, t^{\prime \prime}\right]$; that is, $f_{3} / f_{1}>f_{5} / f_{4}$. If the merger wave is perfectly anticipated, then the proportion of proposed mergers that are approved increases during the period leading up to $t^{\prime}$. During the wave, the proportion of proposed mergers that are approved declines. Finally, at time $t^{\prime \prime}$ the frequency of approved mergers drops to its lowest value. Notice that the proportion of proposed mergers that are approved is also lowest after time $t^{\prime \prime}$.

In words, Proposition 5 implies that the optimal equilibrium amplifies the exogenous variations of a wave of proposed mergers. In Fig. 7, there is $100 \%$ increase in the frequency of proposed mergers between top and bottom of the cycle. However, the difference in terms of approved mergers is much greater. Specifically, in the case of an anticipated merger wave we have $f_{2} / f_{1}>f_{6} / f_{4}$, whereas in the case of an unanticipated merger wave we have $f_{3} / f_{1}>f_{5} / f_{4}$.

In words, the result states that the proportion of approved mergers increases during (and before) an economic boom (which is usually associated to a merger wave). The intuition for this result is that before and during a boom the future expected equilibrium payoff is higher, thus providing greater slack in the no-deviation constraint.

This is only apparently in contradiction with the intuition from Rotemberg and Saloner's (1986) theory of repeated games with fluctuating demand. In their analysis, periods of high demand are typically associated with less efficient collusion. The naive extension would be to expect less efficient compromise when the level of activity is higher. However, the structure of my model is quite different from Rotemberg and Saloner. In my model, a higher activity level corresponds to more frequent interaction, whereas in their model a higher activity level corresponds to greater payoffs today with respect to future payoffs.

\section{Concluding remarks}

I have proposed a repeated-game approach to the problem of international merger policy coordination. Although my analysis is somewhat stylized, it suggests a number of interesting 
results. For example, Proposition 3 suggests that my "mutual concession" equilibrium, as the name suggests, is a two-way street: in order for a country to increase its concession level, it is necessary for the other country to increase its concession level as well.

The foundation of the analysis in this paper is the idea that repeated interaction allows for the self-enforcement of rules that otherwise would not be implementable. This is not a novel idea. Much of the recent trade policy literature is based on the same premise. ${ }^{8}$ What happens when trade policy and merger policy are put together? It is known from game theory that repeated interaction on several strategic variables yields more efficient equilibria than interaction over one variable only. ${ }^{9}$ A number of authors, including Neven and Seabright (1997), Bond (1997), Motta and Onida (1997), Rysman (2000), François and Horn (2000), Richardson (1999), and Horn and Levinsohn (2001) look explicitly at the relation between trade policy and competition policy, including merger policy. However, none of these papers tackles the issue of repeated interaction as indicated above. To my knowledge, Spagnolo (2001) is the first to look at the optimal design of self-enforcing international policy agreements in a multi-issue context. One promising avenue for further research is to study how my results regarding mergers would extend to a multi-issue context, including trade policy and other dimensions of international policy cooperation.

\section{Acknowledgement}

I gratefully acknowledge comments, suggestions and corrections by Mariagiovanna Baccara, Mark Jamieson, Mike Riordan, two anonymous referees, and participants at the International Industrial Organization Society's First Conference. Any remaining errors and deficiencies are my responsibility.

\section{References}

Bacchetta, M., Horn, H., Mavroidis, P., 1997. Do Negative Spillovers, From Nationally Pursued Competition Policies Provide a Case for Multilateral Competition Rules? WTO, Geneva.

Bagwell, Kyle, Staiger, Robert W., 1990. A theory of managed trade. American Economic Review 80, $779-795$.

Barros, Pedro P., Cabral, Luís M.B., 1994. Merger policy in open economies. European Economic Review 38, 1041 - 1055.

Bernhein, Douglas B., Whinston, Michael D., 1990. Multimarket contact and collusive behavior. Rand Journal of Economics 21, 1-26.

Bond, E., 1997. Competition Policy in Customs Unions: A 'Natural Experiment' Using State Level Antitrust Enforcement. Department of Economics, Penn State University.

Cabral, Luís M.B., 2003. International Merger Policy Coordination. Japan and the World Economy 15, $21-30$.

Fox, Eleanor.M., 1998. International Antitrust: Against Minimum Rules; For Cosmopolitan Principles. Antitrust Bulletin 43, 5-13.

François, J., and Horn, H., 2000. Competition Policy in an open economy. Tinbergen Institute Discussion Paper No. TI98 092/2.

Green, Ed., Porter, Robert, 1984. Noncooperative collusion under imperfect price information. Econometrica 52, $87-100$.

Head, Keith, Ries, John, 1997. International mergers and welfare under decentralised competition policy. Canadian Journal of Economics 30, 1104-1123.

Horn, H., Levinsohn, J., 2001. Merger policies and trade liberalisation. Economic Journal 111, 244-276.

Klein, Joel I., 2000. Time for a global competition initiative? Presented at the EC Merger Control 10th Anniversary Conference, Brussels, Belgium.

\footnotetext{
${ }^{8}$ See for example, Bagwell and Staiger (1990).

${ }^{9}$ See, for example, Telser (1980), Bernhein and Whinston (1990), Spagnolo (1999).
} 
Motta, Massimo, Onida, F., 1997. Trade policy and competition policy. Giornale degli Economisti ed Annali di Economia 56, 67-97.

Neven, Damien, Röller, Lars-Hendrik, 2000. The allocation of jurisdiction in international antitrust. European Economic Review 44, 845-855.

Neven, Damien, and Röller, Lars-Hendrik, 2001. "The Scope of Conflict in International Merger Control," University of Lausanne and Wissenschaftszentrum Berlin, February.

Neven, Damien, Seabright, Paul, 1997. Trade Liberalization and the Coordination of Competition Policy. In: Waverman, L., Comanor, W., Goto, A. (Eds.), Competition Policy in the Global Economy: Modalities for Cooperation. Routledge, London.

Neven, Damien, Nuttal, Robin, Seabright, Paul, 1994. Merger in Daylight: The Economics and Politics of Merger Control in the EC. CEPR, London.

Richardson, M., 1999. Trade and competition policies: concordia discors. Oxford Economic Papers 51, $649-664$.

Rysman, Marc, 2000. "Competition Policy in Strategic Trade,” Boston University Industry Studies Program Working Paper No. 100.

Rotemberg, Julio, Saloner, Garth, 1986. A supergame-theoretic model of price wars during booms. American Economic Review 76, 390-407.

Spagnolo, Giancarlo, 1999. On interdependent supergames: multimarket contact, concavity, and collusion. Journal of Economic Theory 89, 127-139.

Spagnolo, Giancarlo, 2001. "Issue Linkage, Credible Delegation, and Policy Cooperation," CEPR Discussion Paper No. 2778, May.

Telser, L.G., 1980. A theory of self-enforcing agreements. Journal of Business 53, 27-44.

Toxvaerd, Flavio, 2002. Strategic Merger Waves: A Theory of Musical Chairs. London Business School. 